\title{
Libertad, propiedad y derecho de cosmopolita: aporías de El Estado comercial cerrado de Fichte
}

Felipe Schwember Augier

\section{(2) OpenEdition \\ Journals}

Edición electrónica

URL: http://journals.openedition.org/ref/640

ISSN: 2258-014X

Editor

EuroPhilosophie Editions

\section{Referencia electrónica}

Felipe Schwember Augier, «Libertad, propiedad y derecho de cosmopolita: aporías de El Estado comercial cerrado de Fichte », Revista de Estud(i)os sobre Fichte [En línea], 11 | 2016, Publicado el 01 junio 2016, consultado el 08 septiembre 2020. URL : http://journals.openedition.org/ref/640

Este documento fue generado automáticamente el 8 septiembre 2020.

(c) EuroPhilosophie 


\title{
Libertad, propiedad y derecho de cosmopolita: aporías de El Estado comercial cerrado de Fichte
}

\author{
Felipe Schwember Augier
}

\section{NOTA DEL AUTOR}

El presente trabajo forma parte del proyecto Fondecyt n⿳o 1160982, titulado "Crítica y recepción de las utopías y del utopismo en el liberalismo libertario y en el liberalismo socialdemócrata del siglo XX: Popper, Hayek, Nozick y Rawls". Agradezco los comentarios de Salvi Turró.

\section{Introducción}

En el entendido de que un sistema jurídico no se encontraba conceptualmente acabado y de que, además, los derechos naturales no serían asegurados a menos que su protección estuviera garantizada a nivel global, los autores de la tradición iusnaturalista solían rematar sus doctrinas del derecho con un capítulo sobre el derecho de gentes o el derecho cosmopolita, según el caso. Tales ramas del derecho se entretejían, en su desarrollo y exposición, con instituciones jurídicas tomadas de otras $\mathrm{y}$, particularmente, con la figura de la propiedad originaria que servía de marco para la justificación de la propiedad.

Esta relación entre propiedad y derecho cosmopolita cobra una peculiar expresión en la filosofía del derecho y en la filosofía política de Fichte, especialmente en su obra El Estado comercial cerrado. Allí la realización y aseguramiento del derecho de propiedad parece desembocar en una verdadera supresión del derecho cosmopolita, entendido como el derecho a recorrer el mundo para ofrecer tratos a los individuos de otros pueblos. De este modo, propiedad y derecho cosmopolita parecen encontrarse en una 
relación inversa, de suerte que el aseguramiento de la primera supone la extinción del segundo, y viceversa.

En lo que sigue se examinarán las razones y alcances de este resultado. Para ello, y después de exponer los argumentos que frecuentemente se daban entre los predecesores de Fichte para delimitar o justificar el derecho cosmopolita y resaltar de este modo las conexiones entre la teoría de la propiedad y derecho cosmopolita en las teorías iusnaturalistas, nos detendremos en la teoría fichteana de la propiedad. Se indagará en aquellos aspectos de la que conducen a una reducción, cuando no a una supresión, del derecho cosmopolita. Se sostendrá que dicha supresión es fruto, fundamentalmente, de la combinación de cinco tesis, tres de las cuales están indisolublemente unidas a la teoría de la propiedad de Fichte: 1) la idea de que la misión primordial del Estado es dar a cada uno lo suyo; 2) la idea de que la propiedad de cada individuo tiene lugar por medio de pactos mutuos; 3) la idea de que la propiedad consiste no en el derecho a una cosa sino en el derecho exclusivo a ejercer una actividad libre.

Se concluirá que, más allá de la paradoja contenida en una propuesta de paz perpetua que culmina con la supresión del derecho cosmopolita, el conflicto entre la distribución equitativa de la propiedad y el derecho a recorrer el mundo abre una serie de interrogantes, tanto con respecto a la relación que guardan en el pensamiento de Fichte derecho y política, como respecto de la relación que tienen entre sí las distintas partes del derecho natural fichteano.

\section{Propiedad, derecho de gentes y derecho cosmopolita}

En su relección De indis prior, Francisco de Vitoria menciona el derecho de comunicación entre los pueblos como uno de los títulos que podrían invocar los españoles para justificar su presencia en América. Más precisamente, dice que "[e]l primer Título puede denominarse «de sociedad y comunicación natural»" (Vitoria 2007, 129). Entre las razones que en seguida Vitoria ofrece en favor de este primer título se encuentra aquella que dice que

"Al principio del mundo, siendo todas las cosas comunes, a cualquiera le estaba permitido dirigirse y recorrer las regiones que quisiera. $\mathrm{Y}$ eso no parece que haya sido abolido por la división de bienes, pues nunca fue intención de las gentes suprimir la intercomunicación de los hombres por ese reparto" (Vitoria 2007, 130).

$\mathrm{Y}$ un poco más adelante añade otro título, relacionado con este que acabamos de citar, y según el cual:

"«Por derecho natural son cosas comunes a todos las corrientes de agua y el mar; también los ríos y los puertos, y a las naves de cualquier parte les es lícito atracar en ellos». Y por la misma razón parecen ser públicos los caminos. Luego nadie puede prohibir su uso. De esto se deduce que los bárbaros harían injuria a los españoles si no les permitiera acceder a su territorio" (Vitoria 2007, 131).

En la formulación de estos títulos, Vitoria saca partido de una de las figuras que autores iusnaturalistas anteriores -como Tomás de Aquino- habían utilizado para justificar la propiedad $^{1}$. Más precisamente, Vitoria extrapola algunas de las consecuencias jurídicas que de dicha figura, la comunidad originaria de bienes, se siguen para el derecho de gentes. Al hacerlo sigue, además, de cerca el razonamiento estándar a favor de la propiedad privada adoptado por los escolásticos. Dicho razonamiento puede reproducirse, groso modo, en los siguientes términos: 
En un principio todas las cosas son comunes y, consecuentemente, todos pueden hacer uso de ellas para conservar la propia vida. Sin embargo, como a fin de cuentas no es posible ni conveniente permanecer en el estado de comunidad originaria, los hombres han convenido en la división de los bienes. Sin embargo, tal división (i.e., la introducción de la propiedad privada) no supone la abolición total de la comunidad originaria de bienes. La abolición de la comunidad originaria es sólo parcial. Ello por varias razones. Primero porque no todos los bienes son susceptibles de división ni lo son, tampoco, bajo cualquier circunstancia. Por su naturaleza no son divisibles aquellos que "el derecho natural ha hecho comunes a todos los hombres", como el mar, los ríos, etc. Estos bienes no son susceptibles de apropiación privada y pueden, por tanto, ser usados siempre por todos los hombres. En segundo lugar la división es parcial porque con ella no se ha querido suprimir el derecho de usar los bienes para sostener la propia vida. Por ese motivo, el que padece una necesidad apremiante siempre puede tomar lo que a otro le sobra para paliar tal necesidad y así salvar la propia vida. De ahí que Tomás de Aquino afirme que no roba ni comete hurto aquel que toma lo que a otro le sobra en estado de necesidad ${ }^{2}$.

Vitoria sigue de cerca este modelo de fundamentación de la propiedad en su desarrollo del derecho de gentes y se aprovecha particularmente de la distinción entre el derecho de uso y el derecho de propiedad sobre los bienes para afirmar que los individuos tienen derecho a recorrer el mundo y a ofrecer tratos de distinto tipo a las gentes de otros pueblos aun cuando, por otra parte, los diversos territorios hayan sido sacados del acervo común al instituirse reinos en ellos.

La persistencia del modelo vitoriano en las teorías iusnaturalistas modernas puede constatarse en el tratamiento de Kant del derecho cosmopolita. Mutatis mutandi, el derecho cosmopolita kantiano recurre, en líneas generales, a los mismo tópicos argumentativos. Por de pronto, el razonamiento que conduce a la deducción de los derechos reales y, de ahí, al derecho cosmopolita de Kant parte también en la comunidad originaria de bienes:

“Todos los hombres están originariamente en posesión común del suelo de la tierra entera (communio fundi originaria), (cada uno) con la voluntad de usarlo, que le corresponde por naturaleza (lex iusti)" (AA VI, 267).

La comunidad originaria de bienes puede inferirse a partir de lo suyo interno de cada uno - a que Kant hace referencia por medio de la lex iust $^{3}$ - y a partir de la forma esférica de la tierra: en la medida en que la tierra no es un plano en el que los hombres puedan dispersarse indefinidamente, y en la medida también en que todos tienen un igual derecho a estar en el mundo, entonces forzosamente habrá que concluir que los hombres están todos originariamente en posesión conjunta de la tierra. Dicha posesión es, en buenas cuentas, condición de posibilidad de cualquier expresión exterior del arbitrio en el mundo sensible. Por eso Kant concluye que la primera adquisición no puede ser sino la del suelo.

Ahora bien, Kant trata la comunidad no como un hecho histórico -en cuyo caso se estaría hablando de una mera invención ${ }^{4}$ - sino como un "concepto práctico de la razón" al que se arriba buscando las condiciones de posibilidad de la coexistencia de los arbitrios en el mundo sensible. Este "concepto práctico" permite establecer las condiciones bajo las cuales son posibles, tanto de la adquisición de una cosa como del ejercicio del derecho de necesidad que limita los efectos de dicha adquisición. Sin entrar en detalles, digamos que la idea de fondo es que la voluntad originaria de cada hombre de usar el suelo y que da origen al concepto práctico de la comunidad originaria de la tierra contiene virtualmente también la única regla conforme a la cual 
es posible el uso simultáneo de la misma según principios jurídicos: dar a cada uno una determinada posesión particular del suelo común (AA VI, 267). Sin una división tal, el uso del suelo, y más precisamente el derecho al uso del suelo, sería un derecho meramente virtual y no devendría nunca en un derecho efectivo (lex iuridica). A su vez, esta división no puede hacerse de tal modo que contravenga la lex iusti que le sirve de base. En consecuencia, no puede ocurrir que, en virtud de la división, alguien quede sin derecho a usar el suelo. El derecho de uso del suelo que se sigue de la lex iusti no es ni puede ser abrogado, por tanto, por la por la instauración de la propiedad.

El razonamiento a favor de la propiedad y de sus límites se puede aplicar analógicamente para establecer, tanto la necesidad de dividir la superficie terrestre en Estados, como el derecho de los individuos a recorrer libremente la tierra. Y eso es precisamente lo que Kant hace. Por eso, al momento de tratar el derecho cosmopolita se refiere nuevamente a la comunidad originaria:

“Todos los pueblos originariamente tienen en común el suelo, pero no están en comunidad jurídica de la posesión (communio) y, por tanto, del uso o de la propiedad del mismo, sino en una comunidad de posible interacción física (commercium), es decir, que se encuentran en una relación universal de uno con todos los demás, que consiste en prestarse a un comercio mutuo, y tienen el derecho de intentarlo, sin que por eso el extranjero esté autorizado a tratarlos como a enemigos" (AA VI, 352, 14-22).

Consciente de los efectos del imperialismo europeo ${ }^{5}$, Kant limita el contenido del derecho cosmopolita a las condiciones de la hospitalidad universal ${ }^{6}$. ¿En qué consisten tales condiciones? Básicamente en el derecho de visita, esto es, el derecho a recorrer el mundo y a proponer "comercio", trato a sus diferentes habitantes, en el más amplio de los sentidos ${ }^{7}$. Este derecho de visita se deriva de la "posesión común de la superficie de la tierra"8 y, a partir de ella, incluso llega Kant a hablar del derecho a hacer uso de la superficie de la tierra (Recht der Oberfläche) ${ }^{9}$.

Tanto el razonamiento de Vitoria como el de Kant ponen de manifiesto las conexiones que existen entre la teoría de la propiedad y la teoría del derecho cosmopolita. En último término, el derecho cosmopolita es levantado en los márgenes del derecho de propiedad, allí donde la apropiación privada no es posible y el derecho de uso, en tanto que remanente irreductible de la comunidad originaria, deja espacio para la interacción común entre los hombres que no son ciudadanos (o súbditos) de un mismo Estado. Así las cosas, el derecho cosmopolita consistirá, fundamentalmente, en el derecho a recorrer el mundo para entrar en tratos con otros y a requerir refugio o asilo cuando la necesidad obligue a ello.

Fichte, por su parte, no hace ni una sola vez mención a la comunidad originaria de bienes, ni en el Fundamento del derecho natural ni en el Estado comercial cerrado. Sin embargo, su filosofía del derecho acusa una relación igualmente estrecha entre la propiedad y el derecho cosmopolita. Su justificación del derecho de propiedad en el derecho originario (Urrecht) y el reconocimiento recíproco tiene enormes y paradójicas repercusiones en las condiciones de ejercicio del derecho cosmopolita, especialmente cuando ellas son examinadas a la luz del proyecto de paz perpetua expuesto en El Estado comercial cerrado ${ }^{10}$.

\section{Propiedad y reconocimiento en Fichte}

Después de deducir el concepto de derecho y de deducir su aplicabilidad - es decir, después de demostrar que el derecho y su aplicación son condiciones de posibilidad del 
arribo a la autoconciencia- Fichte introduce el concepto de "derecho originario". En conformidad con el método trascendental de Fichte que procura determinar la necesidad y el contenido de las instituciones jurídicas por la vía de demostrar que constituyen condiciones de posibilidad de nuestra propia capacidad eficiente libre, el derecho originario es pensado como el conjunto de condiciones bajo las cuales los diferentes agentes pueden ser formalmente libres en el mundo sensible, esto es, como el derecho absoluto de cada persona "a ser únicamente causa en el mundo sensible (y simplemente nunca efecto)" ${ }^{11}$. Por eso, y tal como ocurría con el derecho nativo a la libertad de Kant, el derecho originario de Fichte mienta los derechos fundamentales de la personalidad, el derecho de toda persona a ser tratada como tal, es decir, como un ser capaz de derechos y de obligaciones.

Este derecho impone entonces a todos los demás con los que pudiera entrar en interacción la obligación de no impedir el ejercicio de mi propia causalidad eficiente libre y en consecuencia -y en conformidad con el concepto de reconocimiento (Annerkennung) deducido en la primera sección de libro- la obligación de ejercer su influencia sobre mí sólo a través de conceptos y no a través de la fuerza, al menos en la medida en que yo permita, a su vez, el ejercicio de su causalidad eficiente libre según leyes formales.

La definición y el contenido del concepto de derecho originario expresan, entonces, la posibilidad del ejercicio de la libertad formal de los agentes conforme al principio general del derecho, según el cual el otro también debe poder ser libre a mi lado. Pero ¿qué significa ser solamente causa y nunca efecto en el mundo sensible? Una persona es causa, dice Fichte, cuando realiza en el mundo el fin que se ha dado a sí misma. Eso significa que, en virtud del derecho originario de que cada individuo es titular, cada uno debe ser libre para poder operar en el mundo sensible según el concepto de fin que libremente ha esbozado.

Ahora bien, esto no significa solamente el derecho a la integridad física y a la libertad ambulatoria. Fichte ha demostrado anteriormente que el ejercicio de una acción proyectada sobre un fin libremente esbozado constituye una condición de posibilidad del arribo a la propia autoconciencia. En conformidad con ello, el derecho originario deberá comprender: 1) el derecho a obrar en el mundo sensible; 2) el derecho a la permanencia de dicho mundo, tal como yo lo he conocido y modificado según mi concepto libremente esbozado ${ }^{12}$.

De ahí que no resulte extraño que Fichte afirme que en estas dos condiciones se encuentra "el fundamento de todo derecho de propiedad"13 y que "la parte del mundo sensible conocida por mí y sometida a mis fines, aunque sólo sea en el pensamiento, es originariamente mi propiedad" ${ }^{14}$.

Llegado a este punto no es difícil ver las similitudes con la doctrina de la comunidad originaria: en virtud del derecho originario, todos tienen un igual derecho a obrar sobre todo el mundo sensible y, por lo mismo, el mundo sensible es objeto de una suerte de posesión común. Esto produce un problema porque, obviamente, las esferas de acción de los distintos individuos pueden yuxtaponerse, dando con ello origen a un conflicto acerca del modo en que éstas deben distribuirse.

Los conflictos de este tipo son insolubles en estado de naturaleza fundamentalmente por la imposibilidad de ejercer rectamente el derecho de coacción en estado de naturaleza (imposibilidad de la justicia privada; a esta dificultad además se podría añadir que bien podría ocurrir que ambas partes estuvieran de buena fe). 
Por eso, para evitar esta clase de conflictos es necesario particularizar la esfera de acción de cada individuo, es decir, dividir el mundo sensible en esferas exclusivas de acción. Aquí es donde entra en juego nuevamente el concepto de "reconocimiento recíproco" deducido antes y que sirve de pivote para toda la doctrina del derecho. El reconocimiento recíproco se ha de extender - para evitar ese estado de naturaleza- no solo a la integridad física sino también a la esfera de acción individual exclusiva de cada uno.

"En consecuencia, primero debe cada persona que limitar en general su posesión a un quantum finito del mundo sensible, tan pronto como le es conocida la existencia de una persona exterior a ella. Si quisiera subordinar exclusivamente a sus fines el mundo sensible entero, entonces no podría subsistir a su lado la libertad del otro, que ahora le es bien conocida; pero ésta debe poder subsistir; por tanto, ella [i.e., la persona] está jurídicamente obligada a dejar algo al otro como de su capacidad eficiente libre" (GNR, FW III, pp. 126 - 127 = GA I/3, p. 415).

Un poco más adelante añade:

"Por tanto, su derecho de propiedad (Eigentumsrecht), e.i., el derecho a la posesión exclusiva, se consuma por medio del reconocimiento recíproco (gegenseitige Anerkennung), está condicionado por él y no tiene lugar sin esta condición. Toda propiedad se funda en la reunión de la voluntad de varios en una sola voluntad" (GNR, FW III, p. 129 = GA I/3, p. 417).

Así, y sin necesidad de mencionar si quiera una vez la comunidad originaria, y por medio de la aplicación del concepto de reconocimiento a la justificación de la propiedad, Fichte puede alcanzar casi las mismas conclusiones que hubiera alcanzado de haber partido de ella, a saber, que "toda propiedad se funda en la unificación de la voluntad de varios en una sola voluntad"15.

El instrumento jurídico en que se expresa dicho reconocimiento es el contrato. Concretamente, en lo que Fichte denomina el "contrato de ciudadanía" uno de cuyas cláusulas trata, precisamente, acerca de la instauración y asignación de la propiedad con el fin de delimitar la esfera de acción exclusiva de cada uno, el alcance de la libertad de cada uno y, con ello, los derechos y deberes concretos de cada individuo en particular.

No obstante, al referirse a la necesidad de determinar la "propiedad" de cada cual, Fichte no se está refiriendo solamente a la necesidad de determinar los objetos que han de asignarse a cada individuo sino, en realidad, a un sentido más amplio, "el más amplio" de propiedad, que designa "no sólo, por ejemplo, la posesión de bienes raíces o similares, sino derechos a acciones libres en el mundo sensible en general" 16 .

En el entendido de que la finalidad de la asociación política y del Estado es la propia conservación, la finalidad del contrato de propiedad no será tanto la distribución de cosas como la distribución y división de ciertas actividades o, si se quiere, la distribución pareja de los derechos de uso sobre los bienes externos. Por eso afirma Fichte que "el objeto del contrato de propiedad es una determinada actividad [Tätigkeit]" 17 y no las cosas como tales.

Esta concepción de la propiedad tiene, como veremos, importantes consecuencias para el derecho cosmopolita, consecuencias que son particularmente evidentes en la obra Estado comercial cerrado.

\section{Propiedad y derecho cosmopolita en Fichte}

En el Fundamento del derecho natural, Fichte sostiene que el derecho cosmopolita consiste en el derecho "a circular libremente sobre la superficie de la tierra y a ofrecerse para 
entablar una relación jurídica"18. Este derecho se deriva, explica, del derecho originario del hombre (das ursprüngliche Menschenrecht) que precede a todos los contratos $\mathrm{y}$, al mismo tiempo, los hace posibles: "el derecho a presuponer que todos los hombres pueden establecer con él, mediante contratos una relación jurídica" ${ }^{19}$. Como Kant, Fichte entiende también en esa obra que este derecho de circulación no puede ser denegado arbitrariamente al extranjero, especialmente cuando su conservación está en riesgo ${ }^{20}$. En este sentido, su concepción del derecho cosmopolita no es muy diferente de la concepción kantiana ni, tampoco, de la concepción desarrollada por Vitoria y sus epígonos.

No obstante, este derecho a circular libremente por la superficie de la tierra reconocido en el Fundamento del derecho natural resulta incompatible con el orden jurídico nacional e internacional que Fichte esboza en su trabajo de 1800, el Estado comercial cerrado en la medida en que el mismo es alcanzado - como ya anticipa el título de la obra-mediante el cierre de las fronteras de los distintos estados nacionales al tráfico comercial internacional y tiene por finalidad, por un lado, la consecución de la paz global y, por otro, la realización más perfecta posible de los principios puros del derecho (o, como diríamos hoy, a la realización de la justicia distributiva) a nivel doméstico. En realidad, Fichte cree que lo primero, la paz global, sería es una consecuencia natural de lo segundo, el cierre de las fronteras comerciales de los diferentes estados.

Pero ¿cómo y porqué llega Fichte a esta conclusión? Después de todo, uno podría preguntarse por qué ha de ser necesario suprimir el derecho a recorrer libremente el mundo para alcanzar la realización cabal de la justicia a nivel doméstico y, por añadidura, la paz a nivel internacional?

Creo que se puede responder sumariamente diciendo que por la combinación de cinco factores: 1) La idea de que la misión del Estado, como dice Fichte en el ECC, es dar a cada uno lo suyo y no simplemente asegurarlo; 2) La exigencia de que la determinación de la propiedad de cada cual tenga lugar por medio de declaraciones y acuerdos expresos; 3 ) La idea de que la propiedad consiste no en el derecho a una cosa sino en el derecho exclusivo a una determinada actividad libre; 4) Una teoría objetiva del valor y, 5) La concepción del mercado libre como un estado de anarquía, esto es, de guerra comercial de todos contra todos. Me referiré brevemente a cada uno de estos puntos.

1) La idea de que la misión del Estado es dar a cada uno lo suyo es enunciada por Fichte nada más comenzar el Estado comercial cerrado cuando afirma que: "es misión del Estado, en primer lugar, dar a cada uno lo suyo, ponerlo en su propiedad y, sólo después, protegerlo también" ${ }^{21}$. Evidentemente, la contraposición aquí es con las teorías que admiten la posibilidad de adquirir derechos de propiedad por medio de actos unilaterales (como la prima occupatio o el trabajo) con anterioridad al establecimiento del Estado. Si los derechos de propiedad pudieran ser justificados de esta forma entonces efectivamente el papel del Estado sería el de un mero garante de la propiedad. Fichte desecha rotundamente esta posibilidad: antes del reconocimiento recíproco no hay ni puede haber propiedad y nadie, se entiende, ha renunciado a nada en favor de nadie.

2) De lo anterior se sigue la necesidad de que la propiedad sea establecida mediante pactos. Pero no se trata únicamente de reglas generales acerca de la adquisición de cosas como, por ejemplo, las que formula Nozick ${ }^{22} \mathrm{o}$ las que se encuentran en cualquier derecho civil. En los acuerdos debe quedar inequívocamente claro qué es de cada uno o, mejor, qué debe entenderse en lo sucesivo que es de cada uno, de modo que junto con el 
establecimiento de ciertas reglas formales de adquisición y transmisión de la propiedad, se determina materialmente lo de cada cual.

3) La determinación anterior no se refiere únicamente a la posesión de cosas, sino también a la realización de ciertas actividades o, como también diría Fichte, a un determinado ejercicio de la causalidad eficiente libre ${ }^{23}$. Para estos efectos, el derecho que por otra parte constituye un orden normativo meramente formal- solo puede tener en consideración el único fin que puede presumir en todos los individuos: la autoconservación. Se entiende que todo individuo se pone a sí mismo y, con ello, se pone para todo el futuro. Esto significa que el ejercicio de la actividad libre de cada agente debe poder permitirle su propia conservación, y que consiente en la instauración de un orden jurídico en ese entendido y bajo esa condición. Esto se traduce en la necesidad de consagrar en la constitución del Estado la siguiente regla: cada individuo debe poder vivir de su trabajo. Más precisamente, significa que la propiedad consiste en el derecho a poder vivir del propio trabajo ${ }^{24}$. De ahí que, en virtud de todo lo anterior, los miembros de un Estado dotado de una constitución racional (vernünftigen Staatsverfassung)" ${ }^{25}$ se comprometen es a comprar el trabajo de todos los demás ${ }^{26}$. La contrapartida de esta obligación comunitaria es el deber de ofrecer un servicio que sea demandado. De ahí que bajo una constitución racional los individuos deban reconocer o "conceder" (zugestehen) recíprocamente el derecho a realizar un trabajo. Dicho reconocimiento conlleva o supone, obviamente, el compromiso de comprar a cada uno su trabajo ${ }^{27}$. La otra consecuencia de esta ulterior determinación del derecho de propiedad como el derecho a poder vivir del propio trabajo, es que del derecho a que los demás individuos compren mi trabajo deviene en un derecho de asistencia a mi favor cuando, pese a todo, no obtengo lo suficiente para vivir. Así, y en conformidad con el espíritu general del contrato de propiedad, desde el momento en que un individuo no puede vivir de su trabajo, caducan ipso facto a su respecto las relaciones jurídicas y de propiedad reconocidas hasta ahora y, por tanto, "no está legalmente obligado a reconocer la propiedad de ningún hombre" 28 . Para evitar que la situación de desamparo o de necesidad en que puedan encontrarse algunos derive en la "inseguridad de la propiedad" (Unsicherheit des Eigentums), deben "en conformidad a derecho [Rechtswegen] y como consecuencia del contrato civil, ceder de lo suyo hasta que él pueda vivir" ${ }^{29}$. En consecuencia,

"Cada uno posee su propiedad civil [Bürgereigenthum] sólo en cuanto y bajo la condición de que todos los ciudadanos puedan vivir de la suya; y cesa [la propiedad] en cuanto ellos no pueden vivir y deviene entonces en propiedad de éstos" (GNR, FW III, p. $213=$ GA I/4, pp. 22-23).

Una de las primeras consecuencias del establecimiento de un régimen de propiedad como el descrito, es el carácter público que adquiere el ejercicio de la propia actividad profesional. Dado que todos son, en último término, responsables de la suerte de todos los demás (pues "todos son responsables de que cada uno pueda vivir de su trabajo" ${ }^{30}$ ), el desempeño profesional de cada cual se tornará en un asunto de interés público y no será ya más un asunto meramente privado. De ahí que todos tengan un derecho a controlar o a inspeccionar (Recht der Aufsicht) si cada uno trabaja en su propia esfera tanto como le es necesario para vivir ${ }^{31}$ y que, por su parte, el Estado tenga "necesariamente el derecho a inspeccionar cómo cada uno administra su propiedad de ciudadano (Staatsbürgereigentum)" ${ }^{\prime 32}$.

4) Las facultades fiscalizadoras y reguladoras del Estado fichteano se explican también por su concepción del valor económico de los bienes. Fichte parece creer que una estimación puramente subjetiva del valor de cambio de los bienes propicia, cuando no 
conduce, al estado de depredación mutua que él denomina estado de anarquía comercial:

"El hombre abandonado a sí mismo no valora el producto del otro según el esfuerzo que éste le aplica, sino más bien según el provecho (Nutzen) que piensa sacar de él" (gHS, FW III, p. 454).

Más aún. Podría aventurarse que Fichte considera que cualquier estimación subjetiva del valor de cambio de los bienes tiende a romper la equivalencia de las contraprestaciones y, con ello, la justicia de los intercambios. Al referirse a los intercambios celebrados entre estados comerciales cerrados, dice que entre ellos "no se consideraría la ganancia (Gewinn) sino la igualdad absoluta de valor" ${ }^{133}$. Es difícil no considerar que a esta observación subyace una concepción según la cual los contratos son lucrativos o justos, pero no ambos al mismo tiempo, o no más que de modo accidental. Para que dos personas ganen es necesario que ambas den y reciban lo mismo y "lo mismo" en este contexto no puede hace para Fichte referencia a la estimación que cada uno da sin más a las $\operatorname{cosas}^{34}$. Por eso al momento de examinar el criterio que en primer lugar debe escogerse para determinar el valor (de cambio) de las cosas dice que hay que dejar de lado por el momento la comodidad (Annehmlichkeit) que una cosa produce ${ }^{35}$. Con todo ¿por qué dejar de lado ahora esa comodidad cuando esta bien permitiría explicar la diferencia de valor entre las cosas y, con ello, adoptar un concepto menos estricto de igualdad de las prestaciones?

Tal vez podría argüirse en este sentido que el concepto objetivo del valor que Fichte defiende en el Estado comercial cerrado y el uso sistemático que luego hace de él es coincidente con el fin que el derecho puede atribuirle a los agentes, la autoconservación: el valor de las cosas se estima, en primer lugar, por su aptitud para sostener la vida; el agrado entra en consideración después. $Y$ esa aptitud para sostener la vida es lo que Fichte denomina el valor intrínseco de un bien, y aquel bien que permite sostener la vida por más tiempo vale consecuentemente más que aquel que contribuye a ese mismo fin por menos tiempo. De este modo,

"La medida del valor relativo de las cosas, comparadas entre sí, sería el tiempo durante el cual se podría vivir de ellas" (gHS, FW III, p. 415).

En este sentido, el alimento consumido universalmente, el pan, o en su defecto el material de que está hecho, puede servir de medida del valor de todas las demás cosas, tendría "un valor absoluto, según el cual sería estimado todo otro valor" ${ }^{\text {"36. }}$ Sin embargo, al momento de abordar el problema del valor de los bienes, Fichte ha distinguido entre la medida del valor de aquellas cosas que son necesarias de aquellas que son agradables. Más concretamente ha dicho que: "en el ámbito de la doctrina del derecho, el presunto objetivo de toda actividad libre es la posibilidad y comodidad (Annehmlichkeit) de la vida" ${ }^{37}$. Así, por una parte el pan (o el trigo con el que es hecho) constituye la medida del valor intrínseco de las cosas en tanto es el bien que de modo general es consumido para alimentarse. ¿Cuál es entonces la medida del valor de aquellas cosas que hacen la vida no sólo posible sino también cómoda? La determinación de esta medida se debe hacer, nuevamente, con independencia de los gustos personales (persönlichen Geschmacke) de cada uno ${ }^{38} \mathrm{y}$ vendrá dada por el gasto adicional que, en relación a la fabricación del pan, requiere cada bien que contribuye a la comodidad. o dicho de otro modo, todo bien cuyo valor intrínseco es menor que el del pan es un bien que contribuye a la comodidad ${ }^{39}$. Por lo mismo, el cultivo de tales bienes "no se puede extender más allá de lo que permitan las necesidades de todos, y que nunca deba extenderse tan lejos que alguien carezca por ello de los alimentos imprescindibles" ${ }^{40}$. Así, la teoría del valor de Fichte refuerza la necesidad, ya 
establecida a propósito de la justificación de la propiedad, de regular y controlar la producción e intercambio de los bienes.

5) Como observa Bloch, Fichte descree por completo de la "armonía de los intereses de Smith" (Bloch 2006, 121). Más precisamente, Fichte considera que el estado de anarquía comercial ha dado pábulo a una verdadera guerra comercial de todos contra todos, una "guerra comercial universal pero oculta"41. Esta guerra económica consiste en intentar arrebatarle al otro aquello que tiene bajo su posesión, en obtener ganancias a expensas de otros por el medio que sea o, incluso, y si no hay más remedio, en empeñarse en que al menos mi eventual competidor pierda lo que yo no puedo quedarme para mí. De este modo,

"Al interés por los propios beneficios todavía se une el interés por las pérdidas sufridas por el otro: a veces uno se alegra de poder disfrutar de lo segundo, aún cuando falte lo primero, y ocasiona daño sin beneficio aparente" (gHS, FW III, p. 468).

En este estado de cosas proliferan todo tipo de conductas indecorosas (contrabando soborno, etc.), ideas temerarias como el de la clausura de los mares o los derechos de comercio exclusivo con alguna otra nación (monopolios). Así, el estado de anarquía comercial se desliza inexorablemente hacia el estado de guerra en el sentido estricto del término, con la pauperización y miseria que ello supone para los individuos de los diferentes Estados beligerantes.

Una vez hecha la constatación de que las guerras económicas terminan solapándose o incluso propiciando guerras, la apuesta de Fichte consiste en intentar convencer al lector de la conveniencia de renunciar a ciertos productos suntuarios (como ciertas pieles, la seda, el té, etc.) a cambio de paz.

La consecución de esa apuesta, por otro lado, implica adoptar todas las medidas indicadas en El Estado comercial cerrado. Merced a la concepción de la propiedad de Fichte como el derecho a una cierta actividad libre, estas medidas, entre otras, incluyen o pueden incluir: el intercambio forzoso de mercancías ${ }^{42}$; la limitación del número de individuos que pueden desempeñarse en la industria y, en general, en cada rama de la producción ${ }^{43}$; la reubicación de un individuo en algún sector productivo diferente de aquel en el que se desempeña ${ }^{44}$; la limitación del número de individuos que puedan ejercer como comerciantes ${ }^{45}$; la determinación de los volúmenes de producción necesarios en proporción a la población ${ }^{46}$; la prohibición o la obligación de vender, según el caso ${ }^{47}$; la fijación de precios de acuerdo al valor intrínseco (innerer Wert) de las mercancías ${ }^{48}$ y la emisión de dinero y el control de la masa monetaria circulante ${ }^{49}$. Todas estas medidas tienen por objeto, a fin de cuentas, asegurarse de que el Estado pueda cumplir su misión primordial, esto es, dar y asegurar a que cada uno de sus miembros lo que en derecho le corresponde, su propiedad.

Pero dicho aseguramiento no es posible si, pese a la adopción de todas las medidas indicadas, los nacionales de un Estado aún son libres de intercambiar sus bienes con los nacionales de otro Estado. La posibilidad del comercio internacional puede alterar el equilibrio de los intercambios o -dicho en términos de Nozick- alterar la "pauta" defendida por Fichte según la cual la autoconservación y el mayor nivel de comodidad deben ser conjuntamente posibles para todos y cada uno de los individuos del Estado. Por esa razón, el Estado debe apartar el efecto de la influencia no susceptible de control del extranjero ${ }^{50} \mathrm{y}$ para ello tiene, consecuentemente, el derecho $-\mathrm{y}$, más aún, la obligación- de tornar imposible todo intercambio de los particulares con los extranjeros ${ }^{51}$. 
"Hay que suprimir completamente todo comercio directo del ciudadano con cualquier extranjero [...] Debería, pues, hacerse imposible el comercio directo del ciudadano con cualquier extranjero" (gHS, FW III, p. 484).

Esta prohibición conduce a otra, en la medida en que resulta ser necesaria para asegurar la eficacia del a primera:

"Viajar fuera de un Estado comercial cerrado está reservado para los intelectuales y los técnicos superiores; ya no se permitirá a la ociosa curiosidad ni al afán de diversión llevar de un lado para otro su aburrimiento por todos los países" (gHs, FW III, p. 506).

Pues bien ¿qué ha pasado con el derecho a circular libremente por la superficie de la tierra? Con la necesidad de cerrar comercialmente el Estado arribamos a la creciente supresión del derecho cosmopolita entendido como el derecho a recorrer libremente la superficie de la tierra para ofrecerse a entablar relaciones jurídicas. Dicha supresión comienza con la afirmación de Fichte de que para un estado comercial cerrado el extranjero es prácticamente inexistente ${ }^{52}$ y termina con la prohibición de salir del Estado comercial cerrado. De este modo, el derecho cosmopolita ha desaparecido en virtud de la necesidad de atribuir y garantizar a cada uno lo suyo. Ha desaparecido, al menos para los nacionales del Estado comercial cerrado.

\section{Conclusiones}

El proyecto del Estado comercial cerrado abre una serie de interrogantes tanto, por un lado, por lo que respecta a las relaciones entre el derecho natural (o "derecho racional puro") y la política como, por otro, por lo que se refiere a lugar del derecho cosmopolita dentro del derecho racional puro en general. El tratado de 1796/1797 contemplaba el derecho cosmopolita como parte del derecho natural. Fichte se refería también allí, por otra parte, a la política como "la ciencia que tiene que ver con un Estado particular, determinado por característica contingentes (empíricas) y que considera cuál es la manera más conveniente de realizar en él la ley jurídica" ${ }^{53}$. En El Estado comercial cerrado, en la misma línea, aclara que "la ulterior determinación de las reglas que se ubican en el derecho constitucional puro tiene lugar en la ciencia cuyo concepto defino en lo que sigue, y a la que denomino Política" ${ }^{54}$. Así las cosas, ¿cómo juzgar la desaparición del derecho cosmopolita en El Estado comercial cerrado? ¿Debe interpretarse la misma como una consecuencia de la realización de la mejor forma política atendidas las características de un Estado hic et nunc? En ese caso ¿entran entonces las aspiraciones más altas de la política en contradicción con los principios puros del derecho? Si, por otra parte, no se ve en dicha realización sino el resultado de la instauración de la propiedad tal como Fichte la entiende ¿no entran entonces los principios puros del derecho - la propiedad en particular- en conflicto con el derecho cosmopolita? Y en la medida en que el derecho cosmopolita es una expresión del derecho originario o, en términos kantianos, de la lex iusti ¿cómo debería afrontar un Estado comercial cerrado las demandas de asilo o refugio de los extranjeros? ¿Y la inmigración? Si un Estado comercial cerrado fuese tan exitoso como Fichte espera ¿podría denegar la entrada a los inmigrantes que masivamente llegan de los países que tienen un sistema comercial abierto? ¿Se aplica aquí la regla que propone Fichte para los nacionales de un Estado que padecen necesidad y que dice "no se les debe dejar morir" aun cuando dicha necesidad les sea imputable? ${ }^{55}$

A estas interrogantes se puede sumar aún otra, de carácter exegético, suscitada por la divergencia en el tratamiento del derecho cosmopolita en ambos tratados, el 
Fundamento del derecho natural y El Estado comercial cerrado. Dado que ambos parten del mismo concepto de propiedad y que ambos, empero, arriban a conclusiones diferentes por lo que al derecho cosmopolita concierne ¿cuál debe entenderse es consistente con los principios generales del derecho y, más precisamente, el concepto de propiedad de Fichte? El Estado comercial cerrado se detiene largamente en diversos aspectos de la teoría de la propiedad, que además complementa con ciertas tesis e ideas de carácter económico. No parece aventurado decir que dicha obra no sólo desarrolla más ampliamente los alcances del concepto de propiedad de Fichte sino que también lo hace, por lo que al derecho cosmopolita se refiere, de modo consistente. Si ese es el caso, entonces El Estado comercial cerrado ofrece un modelo de paz perpetua que sólo puede instaurarse, paradójicamente, a expensas del derecho cosmopolita.

\section{Bibliografía}

AQUINO, T. DE. “Suma de teología”. Madrid: Biblioteca de Autores Cristianos, 1990.

BLOCH, E. "El principio esperanza”. Vol. 2. Ed. Francisco Serra. Traducido al español por Felipe González Vicén, Madrid: Editorial Trotta, 2006.

BYRD, B. S. y HRUSCHKA, J. “Kant's Doctrine of Right. A Commentary”. New York: Cambridge University Press, 2010.

FLIKSCHUH, K. y YPI, L. (eds.). “Kant and Colonialism”, Oxford: Oxford Univesity Press, 2014.

FICHTE, J. G. “Fichte-Gesamtausgabe. Bayerischen Akademie der Wissenschaften”. Eds. por Reinhard Lauth, Hans Gliwitzky, Erich Fuchs, Peter K. Schneider y Günter Zöller. Stuttgart-Bad Cannstat: Fromman-Holzboog, 1962-2012.

-. "El Estado comercial cerrado". Traducido por Jaime Franco Barrio. Madrid: Tecnos, 1991.

HUME, D. (2005). “Tratado de la naturaleza humana”. Traducido por Félix Duque. Madrid: Tecnos.

KANT, I. (1999). “La metafísica de las costumbres". Traducido por Adela Cortina Orts y Jesús Conill Sancho. Madrid: Tecnos.

KLEINGELD, P. "Kant and Cosmopolitanism. The Philosophical Ideal of World Citizenship”. Cambridge: Cambridge University Press, 2012.

KLEINGELD, P. (2014). "Kant's Second Thoughts on Colonialism". En Kant and Colonialism, editado por Lea Ypi y Katrin Flikschuh. Oxford: Oxford University Press.

NAKHIMOVSKY, I. (2011). “The Closed Commercial State. Perpetual Peace and Commercial Society from Rousseau to Fichte". New Jersey: Princeton University Press.

NOZICK, R. (1988). “Anarquía, Estado y utopía”. Traducido por Rolando Tamayo. Fondo de Cultura Económica.

RIPSTEIN, A. (2014). «Kant's Juridical Theory of Colonialism.» En Kant and Colonialism, eds. por Lea Ypi y Katrin Flikschuh. Oxford: Oxford University Press.

TIERNEY, BRIAN. (1997). “The Idea of Natural Rights. Studies on Natural Rights, Natural Law, and Church Law 1150 - 1625”. Cambridge: Wm. B. Eerdmans Publishing, 1997. 
VANHAUTE, L. (2014). «Colonist, Traders, or Settlers? Kant on Fair International Trade and Legitimate Settlement.» En Kant and Colonialism, eds. por Lea Ypi y Katrin Flikschuh. Oxford: Oxford University Press.

VITORIA, F. de. (2007). "Sobre el poder civil. Sobre los indios. Sobre el derecho de guerra”. Traducido por Luis Frayle Delgado. Madrid: Tecnos.

YPI, L. “Commerce and Colonialism in Kant's Philosophy of History.” In: Katrin Flikschuh y Lea Ypi (eds.), 2014, 99-126.

\section{NOTAS}

1. Cf. Tomás de Aquino, S.T. II-IIae, q. 66, a. 1 y a. 2. Para los orígenes y función sistemática de la idea de comunidad originaria en la tradición iusnaturalista medieval, cf. Tierney (1997), 131 y ss.

2. Tomás de Aquino, S.T. II-IIae, q. 66, a. 7.

3. Para una explicación de la lex iusti y las demás leyes fundamentales de la doctrina del derecho de Kant, cf. Byrd B. y Hruschka (2010), 44 y ss.

4. Kant, AA VI, 258, 11-21.

5. Con todo, para un examen de la evolución del pensamiento de Kant respecto a la esclavitud, el racismo, el colonialismo y las empresas transcontinentales europeas en general, cf. Kleingeld (2012), 92-123, Kleingeld (2014) y Vanhaute (2014).

6. Como afirma Ripstein, "So, too, in his discussion of why cosmopolitan right must be limited to the mere right of hospitality, he is critical of the expansive scholastic conception of cosmopolitan right as including the right to engage in missionary activities, to construct settlements, and to defend, both when under attack and even prospectively, those activities" Ripstein (2014), 146.

7. Cf., Kleingeld (2012).

8. Kant, AA VIII, 358, 9-10.

9. Kant, AA VIII, 358, 16.

10. Para El Estado comercial cerrado como proyecto de paz perpetua, cf. Nakhimovsky (2011).

11. Fichte, GNR, FW III, p. $113=$ GA I/3, p. 404.

12. Fichte, GNR, FW III, p. $115=$ GA I/3, p. 406.

13. Fichte, GNR, FW III, p. $116=$ GA I/3, p. 407.

14. Fichte, GNR, FW III, p. $116=$ GA I/3, p. 407.

15. Fichte, GNR, FW III, p. $129=$ GA I/3, p. 417.

16. Fichte, GNR, FW III, p. $195=$ GA I/4, p. 8. Énfasis añadido.

17. Fichte, GNR, FW III, p. $210=$ GA I/4, p. 20 .

18. Fichte, GNR, FW III, p. $384=$ GA I/4, p. 164 .

19. Fichte, GNR, FW III, p. $383=$ GA I/4, p. 163.

20. Fichte, GNR, FW III, p. 385 = GA I/4, p. 165.

21. Fichte, gHS, FW III, p. 399.

22. Cf. Nozick (1988), pp. 154 y ss. Otro tanto puede decirse de Hume. Cf. Hume (2005), pp. 673-703. 
23. Fichte, gHS, FW III, p. 401.

24. Fichte, GNR, FW III, p. $212=$ GA I/4, p. 22 .

25. Fichte, GNR, FW III, p. $212=$ GA I/4, p. 22 .

26. Fichte, GNR, FW III, pp. 212 - $213=$ GA I/4, p.22.

27. Fichte, GNR, FW III, p. $213=$ GA I/4, p.22.

28. Fichte, GNR, FW III, p. $213=$ GA I/4, p. 22.

29. Fichte, GNR, FW III, p. $213=$ GA I/4, p. 22.

30. Fichte, GNR, FW III, p. $214=$ GA I/4, p. 23.

31. Fichte, GNR, FW III, p. $214=$ GA I/4, p. 23.

32. Fichte, GNR, FW III, p. $214=$ GA I/4, p. 23.

33. Fichte, GNR, FW III, p. 505.

34. Compárese en cambio la posición de Nozick: "Opiniones anteriores han sostenido que tiene que haber cierto tipo de igualdad entre los bienes que las personas están dispuestas a intercambiar. Porque de otra manera, se pensaba, una parte serla el perdedor. En respuesta, los economistas señalan que los intercambios mutuamente ventajosos requieren únicamente preferencias opuestas. Si una persona prefiere tener el bien del otro a tener el suyo y si, similarmente, la otra persona prefiere tener el bien del primero al suyo propio, entonces un intercambio puede beneficiar a ambos. Ninguno perderá, aun cuando no exista nada por lo que se pueda predicar que sus bienes sean iguales". (1988, p. 72, nota al pie)"

35. Fichte, gHS, FW III, p. 415.

36. Fichte, gHS, FW III, p. 416.

37. Fichte, gHS, FW III, p. 415.

38. Fichte, gHS, FW III, p. 417.

39. Fichte, gHS, FW III, p. 417.

40. Fichte, gHS, FW III, p. 417.

41. Fichte, gHS, FW III, p. 486.

42. Fichte, gHS, FW III, pp. 404-405.

43. Fichte, gHS, FW III, p. 408.

44. Fichte, gHS, FW III, p. 409.

45. Fichte, gHS, FW III, p. 411.

46. Fichte, gHS, FW III, pp. 412 y ss.

47. Fichte, gHS, FW III, pp. 412 y ss.

48. Fichte, gHS, FW III, pp. 415 y ss.

49. Fichte, gHS, FW III, pp. 431 y ss.

50. Fichte, gHS, FW III, p. 421.

51. Fichte, gHS, FW III, p. 419.

52. Fichte, gHS, FW III, p. 433.

53. Fichte, GNR, FW III, p. $286=$ GA I/4, p. 80 .

54. Fichte, gHS, FW III, pp. 390-391.

55. Fichte, GNR, FW III, p. 214 = GA I/4, p. 23 . 


\section{RESÚMENES}

The Closed Commercial State defends a perpetual peace project which, paradoxically, requires the elimination of cosmopolitan law, in other words, the right to freely roam the surface of the earth to offer others to get into legal relations. In this paper it will be argued that this result reflects, fundamentally, Fichte's conception of property. Additionally, certain paradoxes and difficulties that for the whole of Fichte's thought raise this result will be highlighted.

\section{ÍNDICE}

Keywords: Fichte, Property, Cosmopolitan Law, Natural Rights

\section{AUTOR}

\section{FELIPE SCHWEMBER AUGIER}

Universidad Adolfo Ibáñez 